\title{
Efficient Exact Similarity Searches using Multiple Token Orderings
}

\author{
Jongik Kim ${ }^{\# 1}$, Hongrae Lee ${ }^{* 2}$ \\ \# Division of Computer Science \& Engineering, Chonbuk National University \\ 567 Baekje-daero, Jeonju, Republic of Korea \\ ${ }^{1}$ jongik@jbnu.ac.kr \\ ${ }^{*}$ Google Inc. \\ 1600 Amphitheatre Parkway, Mountain View, CA 94043 \\ ${ }^{2}$ hrlee@google.com
}

\begin{abstract}
Similarity searches are essential in many applications including data cleaning and near duplicate detection. Many similarity search algorithms first generate candidate records, and then identify true matches among them. A major focus of those algorithms has been on how to reduce the number of candidate records in the early stage of similarity query processing. One of the most commonly used techniques to reduce the candidate size is the prefix filtering principle, which exploits the document frequency ordering of tokens. In this paper, we propose a novel partitioning technique that considers multiple token orderings based on token co-occurrence statistics. Experimental results show that the proposed technique is effective in reducing the number of candidate records and as a result improves the performance of existing algorithms significantly.
\end{abstract}

\section{INTRODUCTION}

Given a query record and a similarity threshold for a similarity measure, similarity search is to find all records in a collection of records that are similar to the query record. Similarity search is an operation that is essential in a wide range of applications including data cleaning [7], record linkage [9], query refinement [14], and near duplicate detection [19]. Naturally, there have been growing interests in developing efficient algorithms for processing similarity queries.

Similarity query processing is often performed in two phases. The first phase is a filtering phase, where candidate records that are more likely to satisfy the query condition are selected. In the second phase, each record in the candidate list is verified if it is a true match. The first phase is crucial since it has to quickly generate a candidate list that is as small as possible. Thus, a majority of efforts have been on developing efficient filtering techniques.

An important technique commonly used in the first phase is the prefix filtering principle [7]. Assuming a global token ordering, its key intuition is that if two records have a certain number of common tokens then their prefixes should share at least one token. Many algorithms based on the prefix filtering have been proposed (e.g. [7], [10], [15], [17], [19]). Most of them make use of an inverted index built on tokens, where each token is associated with an inverted list, which is a list of ids of records that contain the token. Given a query, a lower bound of the prefix size of the query record can often be determined by exploiting a similarity function definition [1], [15], [17]. Then, a candidate list can be generated by merging inverted lists of tokens in the prefix of the query record.

A widely used technique to reduce the size of a candidate list is to use document frequency ordering of tokens, where the document frequency of a token is the number of records that contain the token. The idea is that if we use an ascending order of document frequencies of tokens, the sizes of inverted lists of tokens in the prefix of a query are minimized. To further reduce the number of candidates, the main focus of recent work (e.g. [17], [19]) has been on tightening the lower bound of the prefix size of a query.

In this paper, we study multiple token orderings to reduce the number of candidates in similarity query processing. We show that a careful partitioning of a collection of records can result in different document frequency orderings among partitions in a way that the number of candidates decreases. We partition a collection of records based on an analysis on the distribution of tokens in both the collection and a query workload. We make the following contributions in the paper:

- We observe that we can partition a collection of records to make partitions have different token orderings, and multiple token orderings can offer benefits in similarity searches. We generalize our observation in the concept of pivot set, and show that we can partition a collection of records by partitioning a pivot set.

- We develop a cost model that measures the effectiveness of alternative pivot set partitionings and propose a partitioning algorithm based on the cost model.

- We experimentally show that the proposed technique substantially reduces the number of candidates and improves existing algorithms by an order of magnitude.

The rest of the paper is organized as follows: Section II provides background on similarity search algorithms and related work and Section III presents a similarity search algorithm used in this paper. Section IV illustrates how a partitioning can reduce the number of candidates and introduces the concept of a pivot set. A cost-based partitioning algorithm that uses pivot 
sets is proposed in Section V. Section VI presents experimental results and Section VII concludes the paper.

\section{PREliminaries AND RELATED Work}

\section{A. Similarity Search Problem}

We define a record as an ordered set of tokens. Given two records $x$ and $y$, we define their overlap similarity to be $|x \cap y|$, the number of overlapping tokens between $x$ and $y$. Given a collection of records $C$ and a query record $x$ with an overlap threshold $\delta$, the similarity search problem is to find every record $y \in C$ such that $|x \cap y| \geq \delta$.

Similarity searches are often performed on a collection of strings, and thus we also consider handling string data. For supporting similarity searches on a collection of strings, we map a string to a record by tokenizing the string into q-grams. A q-gram of a string $s$ is a substring of $s$ of length $q . s$ can be converted into a record of q-gram tokens by sliding a window of length $q$ over the characters of $s$. For example, "string" could be treated as a record of 3-gram tokens \{str,tri,rin, ing . We refer to a record corresponding to a string $s$ as $\operatorname{record}(s, q)$, where $q$ is the gram length. Hence, the overlap similarity between two string $s_{1}$ and $s_{2}$ is defined as $\left|\operatorname{record}\left(s_{1}, q\right) \cap \operatorname{record}\left(s_{2}, q\right)\right|$.

\section{B. Similarity Search using Prefix Filtering}

Given a collection of records $C$ and a query record $x$ with an overlap threshold $\delta$, we can perform the similarity search for $x$ on $C$ using an inverted index that maps each token in $C$ to its corresponding inverted list. An inverted list of a token is a list of ids of records that contain the token. Given an inverted index built on $C$, we first look up the index for each token in $x$ and retrieve the corresponding inverted list if it exists. Then, we merge the inverted lists to count the number of occurrences of each record id in the lists. We finally select those record ids that appear at least $\delta$ inverted lists.

An important technique used in many algorithms for processing similarity queries is the prefix filtering principle [7]. The basic idea is that if two records sorted by a global token ordering have enough overlaps, their prefixes should share at least one token. We first introduce two definitions and then formally state the prefix filtering principle in Lemma 1.

Definition 1: Given an overlap threshold $\delta$ and a record $x$, the prefix of $x$, denoted by $\operatorname{prefix}_{\delta}(x)$, is the set containing the first $|x|-\delta+1$ tokens of $x$ and the suffix of $x$, denoted by $\operatorname{suffix}_{\delta}(x)$, is $x-\operatorname{prefix}_{\delta}(x)$.

Definition 2: Given a collection of records $C, d f_{C}(w)$ denotes the document frequency of a token $w$, which is the number of records containing $w$. The document frequency ordering $\mathcal{O}_{d f}(C)$ denotes the arrangement of tokens in ascending order of their document frequencies in $C$.

Lemma 1: Consider a collection of records $C$ and a query record $x$, where each record $y \in C$ and the query record $x$ are sorted by $\mathcal{O}_{d f}(C)$. Given an overlap threshold $\delta$, if $|x \cap y| \geq \delta$ then $\operatorname{prefix}_{\delta}(x)$ and $\operatorname{prefix}_{\delta}(y)$ must share at least one token.

Example 1: Given a collection of record $C$, consider a query record $x=\left\{w_{A}, w_{B}\right\}$ and a record $y=\left\{w_{B}, w_{C}, w_{D}\right\}$ in $C$ and assume that $x$ and $y$ are sorted by $\mathcal{O}_{d f}(C)$. For an overlap threshold $\delta=2, y$ does not meet the threshold because $\operatorname{prefix}_{\delta}(x)=\left\{w_{A}\right\}$ and $\operatorname{prefix}_{\delta}(y)=\left\{w_{B}, w_{C}\right\}$ do not have a common token. Therefore, we can safely filter $y$ out without seeing any tokens in $\operatorname{suffix}_{\delta}(x)$ and $\operatorname{suffix}_{\delta}(y)$.

Let the prefix lists of $x$ denote the inverted lists associated with the tokens in $\operatorname{prefix}_{\delta}(x)$ and the suffix lists of $x$ denote the inverted lists associated with the tokens in $\operatorname{suffix}_{\delta}(x)$. We can perform a similarity search using the prefix filtering as follows. We retrieve the prefix lists of $x$ and merge them into a candidate list. If a record $y$ is not contained in the candidate list, $y$ cannot meet the overlap threshold by Lemma 1. By sorting $x$ according to the document frequency ordering, we can minimize the number of ids in the candidate list of $x$ because lower frequency tokens are contained in $\operatorname{prefix}_{\delta}(x)$. Since the prefix filtering is a necessary condition and not a sufficient one, we need to verify if each candidate is a true match. The number of occurrences of each candidate $y$ can be counted while merging the prefix lists of $x$. Hence, we continue to count the number of occurrences of $y$ through searching the suffix lists of $x$. A candidate $y$ is a true match if the number of occurrences of $y$ is not less than $\delta$.

\section{Other Similarity Measures and Filtering Methods}

There are several commonly used similarity measures developed for finding similar records. In this paper, we consider four similarity measures listed below. Given a similarity measure, a threshold between two records $x$ and $y$ can be transformed into an equivalent or weaker form of an overlap threshold as follows [1], [15], [17].

- Jaccard similarity between two records $x$ and $y$ is defined as $\frac{|x \cap y|}{|x \cup y|}$. A Jaccard threshold $t$ between $x$ and $y$ is equivalently converted into an overlap threshold $\frac{t}{1+t}(|x|+|y|)$.

- Cosine similarity between two records $x$ and $y$ is defined as $\frac{|x \cap y|}{\sqrt{|x||y|}}$. A cosine threshold $t$ between $x$ and $y$ is equivalently converted into an overlap threshold $t \sqrt{|x||y|}$.

- Dice similarity between two records $x$ and $y$ is defined as $\frac{2|x \cap y|}{|x|+|y|}$. A dice threshold $t$ between $x$ and $y$ is equivalently converted into an overlap threshold $\frac{t(|x|+|y|)}{2}$.

- Edit distance between two strings $s_{1}$ and $s_{2}$ is defined as the minimum number of edit operations to convert $s_{1}$ into $s_{2}$, where an edit operation is insertion, deletion, or substitution of a character. An edit distance $t$ between $s_{1}$ and $s_{2}$ is converted into a weaker form of an overlap threshold max $\left(\left|r e c o r d\left(s_{1}, q\right)\right|,\left|\operatorname{record}\left(s_{2}, q\right)\right|\right)-q \cdot t$. Because the overlap threshold is weaker one, we need post-processing to remove false positives. We exclude the post-processing from our scope. 
Given a collection of records $C$, consider a similarity measure sim other than the overlap similarity, a query record $x$, and a similarity threshold $t$. To generate a candidate list using the prefix filtering, we convert the similarity threshold $t$ into an overlap threshold, identify the size of the prefix of $x$ using the overlap threshold, and retrieve and merge the prefix lists of $x$. The problem here is that we need two records along with $t$ to calculate the overlap threshold but we have only one record $x$. To overcome this problem, we can derive an overlap lower bound $\delta$ of $t$ such that $\forall y, \operatorname{sim}(x, y) \geq t \Longrightarrow|x \cap y| \geq \delta$. The following table shows the overlap lower bound for each similarity measure [1], [15], [17].

\begin{tabular}{c|cccc}
\hline $\operatorname{sim}$ & Jaccard & Cosine & Dice & Edit Distance \\
\hline$\delta$ & $t|x|$ & $t^{2}|x|$ & $\frac{t|x|}{2-t}$ & $\mid$ record $\left(s_{1}, q\right) \mid-q \cdot t$ \\
\hline
\end{tabular}

There is another widely used filtering method called size filtering [1], [2], which is simple but very useful to reduce the number of candidate records. While merging the prefix lists of $x$ to generate candidates, we can prune record ids in each prefix list by exploiting the similarity threshold $t$ and the size of $x$. For example, given a query "string" with edit distance 2, we can prune strings whose sizes are less than 4 or greater than 8 . The following table summarizes the minimum and maximum size bounds for each similarity measure.

\begin{tabular}{c|cccc}
\hline sim & Jaccard & Cosine & Dice & Edit Distance \\
\hline min_size & $t|x|$ & $t^{2}|x|$ & $\frac{t|x|}{2-t}$ & $\left|s_{1}\right|-t$ \\
\hline max_size & $\frac{|x|}{t}$ & $\frac{|x|}{t^{2}}$ & $\frac{(2-t)|x|}{t}$ & $\left|s_{1}\right|+t$ \\
\hline
\end{tabular}

To exploit the size filtering, we sort records in $C$ in ascending order of their sizes and assign a sequential id to each record in $C$. When building the inverted index of $C$, we make each inverted list sorted in ascending order of record ids. While scanning each inverted list, we can effectively skip ids of records whose sizes are less than min_size and stop scanning as soon as we encounter the id of a record whose size is larger than $\max \_s i z e .{ }^{1}$ We use the size filtering as well as the prefix filtering in this paper.

\section{Related Work}

Set similarity join is the problem of finding similar pairs of records from two collections of records, which is essential in many applications including data cleaning [7] and near duplicate detection [19]. Many algorithms are developed for the problem of set similarity join [1], [2], [5], [7], [8], [13], [15], [16], [17], [18], [19]. Most of these algorithms can be applied to the search problem in their original form or with slight modification. Several algorithms and inverted index structures have been proposed for the similarity search problem [3], [4], [5], [6], [10], [11], [12], [20].

The proposed technique in this paper is orthogonal to specific heuristics of similarity search algorithms, and can benefit a wide range of algorithms that are based on the prefix filtering principle.

\footnotetext{
${ }^{1}$ An inverted list is usually implemented by an array and skipping can be implemented by a binary search on an inverted list.
}

\section{SEARCh Algorithm}

In this section, we present a similarity search algorithm, which is a straight extension of existing algorithms for handling partitioned collections of records. We make the following assumptions in the algorithm.

- A collection of records $C$ have been already partitioned.

- The inverted list of each token $w$ is partitioned into an array of inverted lists, denoted by $I_{w}$, such that $I_{w}[P]$ is the inverted list of $w$ in a partition $P$.

- For a query record $x$, inverted lists of tokens in $x$ have been identified.

Algorithm 1 shows an overview of our similarity search algorithm. The algorithm performs a similarity search on each partition and takes the union of results. In the algorithm, lowerbound $(|x|, t)$ returns the overlap lower bound of $t$ and tightbound $(|x|,|y|, t)$ returns the overlap threshold corresponding to $t$ (see Section II(C)).

Let the token dictionary $\mathcal{D}$ of a collection of records $C$ is a set of tokens contained in $C$, that is, $\mathcal{D}=\bigcup_{r \in C} r$. Because not all tokens in a query record $x$ are necessarily contained in the token dictionary of $C, x^{\prime}=x \cap \mathcal{D}$ instead of $x$ can be used for the prefix filtering. The algorithm first calculates the prefix size $p_{x^{\prime}}$ of $x^{\prime}=x \cap \mathcal{D}$ (Lines 2 and 3). In each partition (Line 4), the algorithm generates candidates using the prefix filtering and size filtering (Lines 5-9) and verifies if each candidate is a true match (Lines 10-17). The details of the algorithm are as follows.

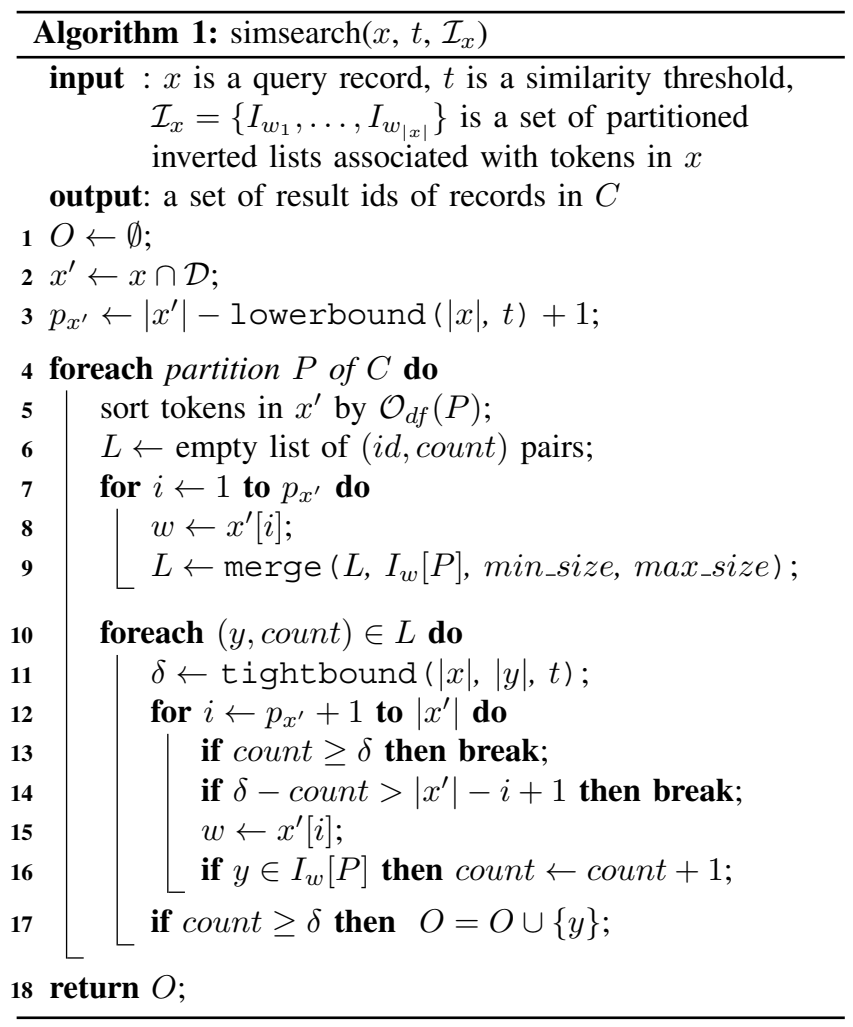


Candidate Generation: Since the document frequency of a token can differ from partition to partition, Algorithm 1 sorts tokens in $x^{\prime}$ in each partition to identify the prefix of $x^{\prime}$ (Line 5). The algorithm sequentially accumulates each prefix list to a candidate list $L$, which is initially empty (Lines 6-9). Although it is theoretically inferior to merge the prefix lists one by one, the algorithm exhibits good spatial locality and as a result merges the prefix lists very fast. We name this algorithm SeqMerge. The algorithm also uses the size filtering while merging the prefix lists (Line 9). We can derive several variants of this algorithm by applying different merging techniques as follows.

- MergeOpt [15] uses a min-heap to efficiently merge the prefix lists (Lines 6-9).

- DivideSkip [10] aims to reduce the number of candidates by extending the size of the prefix to $\left|x^{\prime}\right|-\delta+c$, where $c \geq 1$ (Line 3). It also merges the prefix lists using a min-heap based algorithm, MergeSkip [10] (Lines 6-9).

- PPMerge, which is named after ppjoin [19], exploits the positional filter [19] after tightening the overlap threshold for each candidate record (Line 9).

- EDMerge, which is applicable to the edit distance only, reduces the prefix size using the mismatch filter proposed in Ed-join [17] (Line 3).

Verification: For each candidate, ( $i d$, count), identified in the prefix lists, Algorithm 1 increases count whenever id appears in a suffix list (Line 16). The algorithm stops searching suffix lists as soon as count reaches the overlap threshold $\delta$ (Line 13). It also stops searching if $\delta$-count is greater than the number of remaining suffix lists (Line 14), that is, the current id cannot meet the threshold $\delta$ even if the id appears in all the remaining lists. We call such stoppings early termination. The algorithm uses a binary search for searching each suffix list (Line 16). Because record ids in each inverted list and the candidate list are sorted in the order of their values, the algorithm can reduce the search range of each suffix list every time a candidate searches the suffix list.

\section{Multiple Token Orderings}

In this section, we introduce an example of multiple token ordering by partitioning a collection of records and show how it can benefit similarity search. We also define pivot sets by which we partition a collection of records and implement multiple token orderings that can reduce the candidate size.

\section{A. Motivating Example}

For the example collection of strings $C$ depicted in Figure 1(a), consider a query record $x=\operatorname{record}(" r e a b y ", 2)$ $=\{r e, e a, a b, b y\}$ and an overlap threshold $\delta=2{ }^{2}$ We use the following gram-to-token mapping table for the sake of presentation.

\footnotetext{
${ }^{2}$ The overlap threshold $\delta=2$ can be derived from edit distance 1 or Jaccard
} threshold 0.5 (see Section II(C))

\begin{tabular}{c|ccccccc}
\hline token & $\boldsymbol{w}_{1}$ & $w_{2}$ & $\boldsymbol{w}_{3}$ & $w_{4}$ & $w_{5}$ & $w_{6}$ & $\ldots$ \\
\hline gram & ea & er & re & le & cl & he & $\ldots$ \\
\hline \hline$d f_{C}$ & $\mathbf{6}$ & 5 & $\mathbf{5}$ & 4 & 4 & 4 & $\ldots$ \\
\hline
\end{tabular}

To exploit the prefix filtering, we identify $x^{\prime}=x \cap \mathcal{D}=$ $\left\{w_{1}, w_{3}\right\}$, where $\mathcal{D}$ is the token dictionary of $C$ (see Section III). The following examples illustrate the processing of the query in unpartitioned case and partitioned case.

Example 2: We can evaluate the query on the collection $C$ depicted in Figure 1(a) as follows. We first identify that $\operatorname{prefix}_{\delta}\left(x^{\prime}\right)=\left\{w_{3}\right\}$ and $\operatorname{suffix}_{\delta}\left(x^{\prime}\right)=\left\{w_{1}\right\}$ since $d f_{C}\left(w_{3}\right)<d f_{C}\left(w_{1}\right)$. We then generate the candidate list $\left[r_{1}, r_{2}, r_{3}, r_{5}, r_{7}\right]$ from the prefix list $I_{w_{3}}[C]$ (refer to Figure 1(b) or (c)). For each record in the candidate list, we search for its occurrence in the suffix list $I_{w_{1}}[C]$, and finally obtain $\left\{r_{1}, r_{2}\right\}$.

Example 3: Suppose we partition $C$ into $P_{1}$ and $P_{2}$ as shown in Figure 1(b). We can evaluate the query on each partition and take union of the results. In $P_{1}$, the candidate list $\left[r_{1}, r_{2}\right]$ is generated from the prefix list $I_{w_{1}}\left[P_{1}\right]$ and $\left\{r_{1}, r_{2}\right\}$ is returned after searching $I_{w_{3}}\left[P_{1}\right]$ for each candidate. In $P_{2}$, the prefix list $I_{w_{3}}\left[P_{2}\right]$ is empty and there is no result of the query. Hence, the result of the query is $\left\{r_{1}, r_{2}\right\}$.

Let us consider query processing costs of the two examples above. We compute the cost of evaluating a query as the sum of cost for merging the prefix lists, no. of candidates $\times$ cost for binary searches on the suffix lists, and cost for sorting the query record. Hence, costs for evaluating $x$ on $C, P_{1}$, and $P_{2}$ are as follows.

$$
\begin{aligned}
& \text { In } C: \begin{aligned}
& \left|I_{w_{3}}[C]\right|+\left|I_{w_{3}}[C]\right| \times\left\lceil\log \left|I_{w_{1}}[C]\right|\right\rceil+\left|x^{\prime}\right| \log \left|x^{\prime}\right| \\
& =5+5 \times\lceil\log 6\rceil+2 \log 2=22 \\
\text { In } P_{1}: & \left|I_{w_{1}}\left[P_{1}\right]\right|+\left|I_{w_{1}}\left[P_{1}\right]\right| \times\left\lceil\log \left|I_{w_{3}}\left[P_{1}\right]\right|\right\rceil+\left|x^{\prime}\right| \log \left|x^{\prime}\right| \\
& =2+2 \times\lceil\log 5\rceil+2 \log 2=10 \\
\text { In } P_{2}: & \left|I_{w_{3}}\left[P_{2}\right]\right|+\left|I_{w_{3}}\left[P_{2}\right]\right| \times \log \left|I_{w_{1}}\left[P_{2}\right]\right|+\left|x^{\prime}\right| \log \left|x^{\prime}\right| \\
& =0+0 \times \log 4+2 \log 2=2
\end{aligned}
\end{aligned}
$$

The total number of candidates in the unpartitioned case is 5 while that of the partitioned case is $2\left(P_{1}: 2+P_{2}: 0\right)$. This is because $P_{1}$ and $P_{2}$ have different token orderings on $w_{1}$ and $w_{3}$. As a result, we can reduce the cost for evaluating the

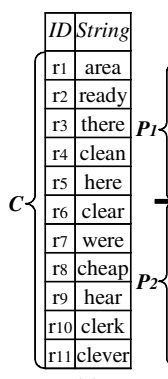

(a) (b)

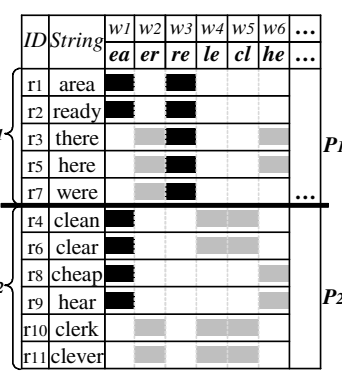

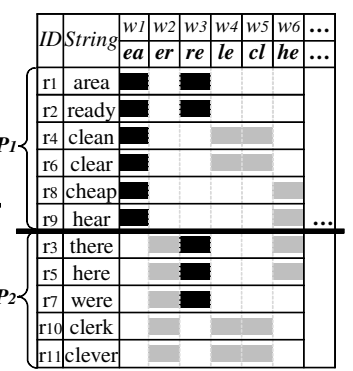

(c)
Fig. 1. Motivating example 
query by $22-(10+2)=10$ through the partitioning.

Consider an alternative example of partitioning depicted in Figure 1(c). With the partitioning, we can compute the cost for evaluating $x$ as $\left\{P_{1}=2+2 \times\lceil\log 6\rceil+2 \log 2=10\right\}+\left\{P_{2}=\right.$ $0+0 \times\lceil\log 3\rceil+2 \log 2=2\}=12$, which is the same as that of the partitioning depicted in Figure 1(b).

\section{B. Where the Benefit Comes from}

From the partitioning example in Figure 1(b), we observe that records that contain $w_{3}$ are grouped together into $P_{1}$. Likewise, in the example depicted in Figure 1(c), records contain $w_{1}$ are grouped together into $P_{1}$. In both examples, grouping records that share a token in $x$ into a partition results in the change on the token order of $w_{1}$ and $w_{3}$ between $P_{1}$ and $P_{2}$. The different token orderings between two partitions make the number of candidates of the query reduced. We formalize our observation as follows.

Definition 3: Given a collection of records $C$ and a token $w, R(w)$ denotes the set of records in $C$ which contain $w$.

Lemma 2: Given a collection of records $C$ and a query record $x$ with an overlap threshold $\delta$, consider two tokens $w_{p} \in$ $\operatorname{prefix}_{\delta}(x)$ and $w_{s} \in \operatorname{suffix}_{\delta}(x)$ such that $R\left(w_{p}\right) \neq \emptyset$, $R\left(w_{s}\right) \neq \emptyset$, and $R\left(w_{p}\right) \not \subset R\left(w_{s}\right)$. We can reduce the number of candidates if we partition $C$ as follows.

A. If we divide $C$ by grouping records in $R\left(w_{p}\right)$ into a partition $P$, the token order of $w_{p}$ and $w_{s}$ is changed in $P$ and the number of candidates is reduced by at least $\left|R\left(w_{p}\right)\right|-\left|R\left(w_{s}\right) \cap P\right|$.

B. If we divide $C$ by grouping records in $R\left(w_{s}\right)$ into a partition $P$, the token order of $w_{p}$ and $w_{s}$ is changed in one or more partitions other than $P$ and the number of candidates is reduced by at least $\left|R\left(w_{p}\right)-P\right|$.

Example 4: In Figure 1(b), all records containing $w_{3}$ are grouped into $P_{1}$. As a consequence, the token order of $w_{1}$ and $w_{3}$ is changed in $P_{1}$ from $d f_{C}\left(w_{1}\right)>d f_{C}\left(w_{3}\right)$ to $d f_{P_{1}}\left(w_{3}\right)>d f_{P_{1}}\left(w_{1}\right)$, which in turn causes the prefix and the suffix of $x^{\prime}$ to be exchanged in $P_{1}$. Hence, the number of candidates is reduced by $\left|R\left(w_{3}\right)\right|-\left|R\left(w_{1}\right) \cap P_{1}\right|=5-2=3$.

Example 5: In Figure 1(c), all records containing $w_{1}$ are grouped into $P_{1}$ and the token order of $w_{1}$ and $w_{3}$ is changed in $P_{2}$. As a result, the frequency of the prefix token $w_{1}$ in $P_{2}$ become zero and the number of candidates is reduced by $\left|R\left(w_{3}\right)-P_{1}\right|=\left|\left\{r_{3}, r_{5}, r_{7}\right\}\right|=3$.

The discussion so far has focused on a single query. However, a partitioning that brings benefit to a query may not contribute to another query because queries contain different combinations of tokens. Consider the following example.

Example 6: The partitioning depicted in Figure 1(b) cannot reduce the number of candidates for a query record record("her", 2) $=\left\{w_{2}, w_{6}\right\}$ with an overlap threshold $\delta=2$. This is because the token order of $w_{2}$ and $w_{6}$ is not changed in both $P_{1}$ and $P_{2}$. If we partition $C$ as depicted in Figure 1(c), however, both queries "reaby" and "her" have benefit from the partitioning.

The reason why the partitioning in Figure 1(c) brings benefit to both queries is that grouping $R\left(w_{1}\right)$ into a partition does not affect grouping $R\left(w_{2}\right)$ into another partition (note that $w_{1}$ is contained in "reaby" and $w_{2}$ is contained in "her"). The key insight is that two tokens of $w_{1}$ and $w_{2}$ do not co-occur in any record. By partitioning a collection of records using those tokens that do not co-occur in any record, multiple queries can have benefit from the partitioning. In our example, there are other combinations of tokens that do not co-occur in any record such as $\left\{w_{3}, w_{4}\right\},\left\{w_{3}, w_{5}\right\},\left\{w_{4}, w_{6}\right\}$, and $\left\{w_{5}, w_{6}\right\}$. We call such a combination of tokens a pivot set. A question here is how to select a pivot set that brings benefit to many queries. We postpone the discussion of selecting a good pivot set to Section V(B).

Although partitioning can bring benefit to a query, it introduces extra overhead to sort the query record in each partition. In our example, we need to sort the query record only once when we do not partition the collection of records. However, we have to sort the query record twice if we divide the collection into two partitions to take advantage of multiple token orderings. Hence, we need to adjust the number of partitions to balance the trade-off between the benefit and the overhead of partitioning. We will discuss this issue in Section $\mathrm{V}(\mathrm{C})$ and Section V(D).

\section{Pivot Sets of Partitions}

In this section, we formalize the concept of a pivot set and show how we can partition a collection of records using a pivot set.

Definition 4: A pivot set $S$ is a set of distinct tokens such that $\forall w_{i}, w_{j} \in S, R\left(w_{i}\right) \cap R\left(w_{j}\right)=\emptyset$. A maximal pivot set is a pivot set that is not a subset of any other pivot set.

Example 7: In Figure 1, $S=\left\{w_{1}, w_{2}\right\}$ is a pivot set because there is no record that has both $w_{1}$ and $w_{2} . S$ is a maximal pivot set because if we insert any of tokens in $C$ into $S, S$ is not a pivot set any more.

Definition 5: Given a collection of records, consider a partition $P$ and a pivot set $S$. $S$ determines $P$ if and only if $P$ has all and only those records that contain at least one token in $S$. We also say that $S$ covers the records in $P$ and $S$ is the pivot set of $P$.

Example 8: In Figure 1(c), the pivot set $\left\{w_{1}\right\}$ determines $P_{1}$ because all the records in $P_{1}$ contain $w_{1}$ and any record in $P_{2}$ does not contain $w_{1}$.

Property 1: Consider two partitions $P_{1}$ and $P_{2}$ and their pivot sets $S_{1}$ and $S_{2}$. Because $P_{1} \cap P_{2}=\emptyset, S_{1}$ and $S_{2}$ should 
satisfy that $\forall w_{i} \in S_{1}$ and $\forall w_{j} \in S_{2}, R\left(w_{i}\right) \cap R\left(w_{j}\right)=\emptyset$. Therefore, $S_{1} \cup S_{2}$ is another pivot set.

Given a collection of records, if we find $n$ pivot sets of $\left\{S_{1}, \ldots S_{n}\right\}$ that satisfy Property 1 , we can use the pivot sets to divide the collection into $n$ partitions such that the $i^{\text {th }}$ partition is determined by $S_{i}$. According to Property 1 , $S=\bigcup_{i=1}^{n} S_{i}$ is also a pivot set. Therefore, the problem of finding $n$ pivot sets satisfying Property 1 is equivalent to the problem of finding a pivot set (e.g. $S$ ) and dividing it into $n$ disjoint subsets (e.g. $\left\{S_{1}, \ldots, S_{n}\right\}$ ). Note that each subset is a pivot set by Definition 4 .

Definition 6: An orphan record is a record that cannot be included in any partition because the pivot set used in partitioning contains no token in the record.

Example 9: If we partition the collection of records in Figure 1(a) using the pivot set $\left\{w_{5}, w_{6}\right\}, r_{1}, r_{2}$, and $r_{7}$ become orphan records because these records do not contain any of $w_{5}$ and $w_{6}$.

When there are orphan records after partitioning, we can either assign each of them to a randomly selected partition or make another partition for them.

As a summary of this section, we partition a collection of records by solving the following two subproblems.

1) Given a collection of records, find a good pivot set which is a basis for partitioning.

2) Given a pivot set of a collection of records, find a good partitioning of the pivot set.

There are the following considerations in our formulation of a pivot set.

- For any two tokens, $w_{i}$ and $w_{j}$, we need to know the intersection size between $R\left(w_{i}\right)$ and $R\left(w_{j}\right)$ to construct and utilize pivot sets. We describe details in Section V(A).

- A challenge is to select a good pivot set among many combinations. We show that it is an NP-hard problem under our definition and present a greedy algorithm to choose a good pivot set in Section V(B).

- As we described in Section IV(B), there is a trade-off between the benefit and the overhead of partitioning. We propose a cost-based pivot set partitioning algorithm in Section $\mathrm{V}(\mathrm{C})$ and a cost estimation technique in Section $\mathrm{V}(\mathrm{D})$ to find a partitioning that balances this trade-off.

- One last consideration is the existence of a pivot set that can make enough partitions. For example, the pivot set selected for partitioning can have only a few tokens. We will discuss this issue in Section V(C) and Section V(E).

\section{Pivot Set Partitioning}

\section{A. Token Graph}

In this section, we define the token graph and the disjoint token graph of a collection of records. They are used for pivot set selection, pivot set partitioning, and cost estimation

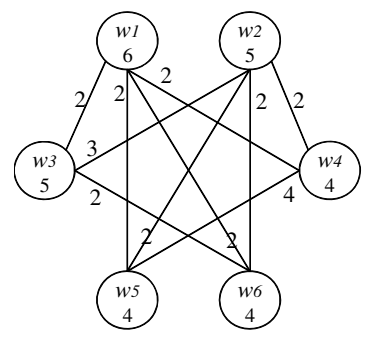

(a)

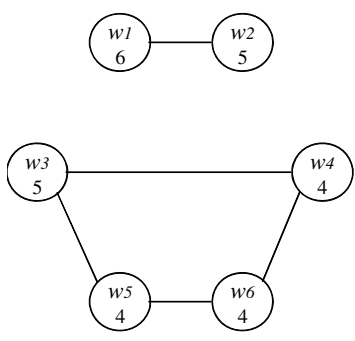

(b)
Fig. 2. Example of token graph

in the later sections.

Definition 7: Given a collection of records $C$, the token graph of $C$ is an undirected graph $G=(V, E)$ such that $V=\{w \mid w$ is a token in $C\}$ and $E=\left\{\left(w_{i}, w_{j}\right) \mid w_{i} \in V\right.$, $w_{j} \in V, R\left(w_{i}\right) \cap R\left(w_{j}\right) \neq \emptyset$, and $\left.i \neq j\right\}$. Each vertex $w$ is weighted with $|R(w)|$, and each edge $\left(w_{i}, w_{j}\right)$ is weighted with $\left|R\left(w_{i}\right) \cap R\left(w_{j}\right)\right|$.

Definition 8: The disjoint token graph $G^{\prime}=\left(V^{\prime}, E^{\prime}\right)$ of a token graph $G=(V, E)$ is an undirected graph such that $V^{\prime}=V$ and $E^{\prime}=\left\{\left(w_{i}, w_{j}\right) \mid w_{i} \in V^{\prime}, w_{j} \in V^{\prime}\right.$, $R\left(w_{i}\right) \cap R\left(w_{j}\right)=\emptyset$, and $\left.i \neq j\right\}$.

Example 10: Figure 2(a) shows the token graph of the collection of records in Figure 1(a). The graph in Figure 2(b) is the disjoint token graph of the token graph in Figure 2(a).

For any two tokens, $w_{i}$ and $w_{j}$, we can easily obtain $\left|R\left(w_{i}\right)\right|,\left|R\left(w_{j}\right)\right|$, and $\left|R\left(w_{i}\right) \cap R\left(w_{j}\right)\right|$ by looking up weights of vertices and edges of the token graph. When we look up the token graph for finding sizes related to a token $w$, the token graph returns 0 if it does not contain $w$.

Given a collection of records $C$, we can build a token graph by scanning $C$ once. Let $n$ be the average number of tokens in a record and $\mathcal{D}$ be the token dictionary of $C$. The time complexity for building a token graph is $O\left(n^{2} \times|C|\right)$ and the space complexity is $O\left(|\mathcal{D}|^{2}\right)$. It is worthwhile to note that a token graph is used only for selecting and partitioning a pivot set and it is removed after the partitioning is done. Therefore, the cost for building a token graph does not affect the cost of evaluating a query. Nonetheless, a naive construction of a token graph may incur too much computational and spatial overhead.

To make a token graph be a compact structure, we can use only a small portion of tokens in $\mathcal{D}$. It is well-know that frequencies of tokens usually follow a Zipf distribution. Taking advantage of the distribution, we can use $k$ distinct tokens such that $\sum_{i=1}^{k}\left|R\left(w_{i}\right)\right| \geq \tau \times \sum_{i=1}^{|\mathcal{D}|}\left|R\left(w_{i}\right)\right|$, where $\tau<1$ and $\left|R\left(w_{i}\right)\right| \geq\left|R\left(w_{j}\right)\right|$ if and only if $i \leq j$. A compact token graph can be built by scanning $C$ twice. We first calculate the weight of each vertex by scanning $C$. We then select $k$ high frequency tokens. We finally calculate the weight of each 
edge only for the selected tokens by scanning $C$ again. Further discussions on the compaction of a token graph are presented in Section VI.

\section{B. A Pivot Set Selection Algorithm}

Given a collection of records $C$, the goodness of a pivot set is clearly dependent on queries issued to $C$. As we described in Section IV(B), queries containing a token $w$ are likely to have benefit when we group records in $R(w)$ together into a partition. Hence, if every query issued to $C$ can find some of its tokens in a pivot set $S$, we may bring benefit to every query by partitioning $C$ using $S$. Based on this intuition, we measure the goodness of a pivot set $S$ by the sum of weights of tokens in $S$, where the weight of a token is the number of queries that contain the token. For simplicity, we assume that the token distribution of queries follows that of $C$. By this assumption, the document frequency of a token is the weight of the token. Thus, the goodness of a pivot set $S$ is measured by $M_{1}=\sum_{w \in S} d f_{C}(w)$. Note that $M_{1}$ also denotes that how many records in $C$ a pivot set covers.

It is obvious that a pivot set is necessarily maximal to maximize $M_{1}$. A maximal pivot set in $C$ is equivalent to a maximal clique in the disjoint token graph of $C$. The problem of selecting an optimal pivot set under $M_{1}$ is identical to the problem of finding a maximum clique in the disjoint token graph, which is an NP hard problem. Hence, we introduce another measure to develop a heuristic for selecting a good pivot set. We select a pivot set $S$ such that for each token $w \in S, M_{2}=d f_{C}(w)$ is high. The intuition is that if we partition $C$ using a token whose weight is high, many queries can have benefit from the partitioning.

Algorithm 2 shows our pivot set selection algorithm. GreedySelection finds a pivot set in a greedy fashion using

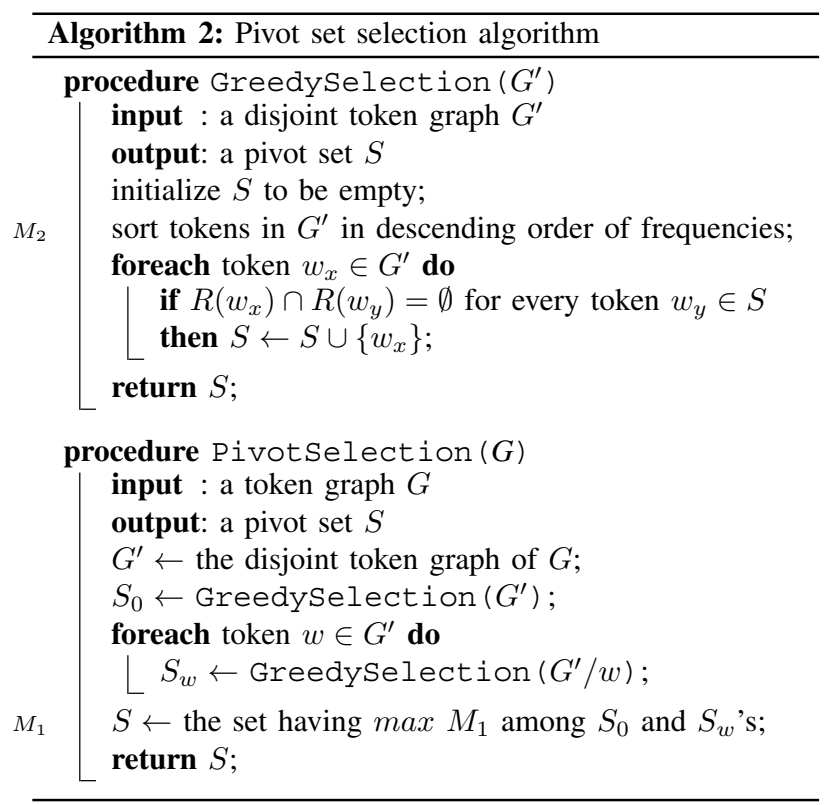

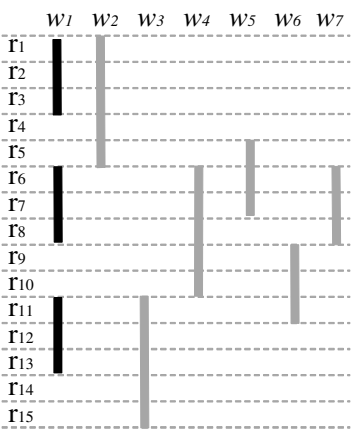

(a)

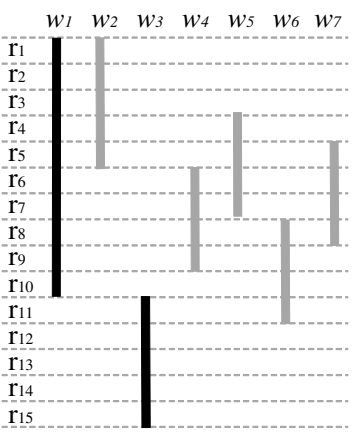

(b)
Fig. 3. Examples of collections of records

$M_{2}$. By sorting tokens in descending order of their document frequencies, the algorithm selects high frequency tokens first. The foreach statement in GreedySelection guarantees that the procedure always makes a maximal pivot set. PivotSelection selects a pivot set using $M_{1}$ after generating multiple pivot sets. In PivotSelection, $G^{\prime} / w$ denotes the graph formed by eliminating a vertex (or a token) $w$ from $G^{\prime}$. Given a disjoint token graph $G^{\prime}$, PivotSelection first finds a pivot set $S_{0}$ using GreedySelection. Then, it finds $\left|G^{\prime}\right|$ more pivot sets by eliminating each vertex from $G^{\prime}$, where $\left|G^{\prime}\right|$ is the number of tokens in $G^{\prime}$. It finally selects a pivot set using $M_{1}$ among $\left|G^{\prime}\right|+1$ pivot sets.

Example 11: For the collection of records in Figure 3(a), if we use GreedySelection only, we select the pivot set $\left\{w_{1}\right\}$. As a result, we can make at most one partition using the pivot set. However, PivotSelection selects a better pivot set of $\left\{w_{2}, w_{3}, w_{4}\right\}$ by considering $\left|G^{\prime}\right|$ more pivot sets.

The measure $M_{2}$ is likely to select a pivot set that contains high frequency tokens. Such a pivot set tends to result in a small number of partitions, where some of which contain most records. Let us consider the following example.

Example 12: For the collection of records in Figure 3(b), PivotSelection selects the pivot set of $\left\{w_{1}, w_{3}\right\}$. It loses the chance to divide $w_{2}$ and $w_{4}$ by selecting the high frequency token $w_{1}$. As a consequence, a large partition of $R\left(w_{1}\right)=\left\{r_{1}, \ldots, r_{10}\right\}$ is created. Because $R\left(w_{1}\right)$ contains all the records in $R\left(w_{2}\right), R\left(w_{4}\right), R\left(w_{5}\right)$, and $R\left(w_{7}\right)$ and most of the records in $R\left(w_{6}\right)$, we cannot have benefit from the partitioning unless queries contain the token $w_{3}$.

Although the algorithm does not select an optimal pivot set, our partitioning algorithm in the next section finds large partitions such as $R\left(w_{1}\right)$ in the example above and divides those partitions recursively using tokens such as $w_{2}$ and $w_{4}$. Hence, the pivot selection algorithm is not very sensitive to the benefit as long as it can find a pivot set that consists of high frequency tokens and covers most records in the collection. 


\section{A Cost-based Partitioning Algorithm}

A naive way to partition a pivot is generating all possible partitionings and choosing the best one. However, the total number of possible partitionings of a pivot set having $n$ tokens is equal to the $n^{t h}$ Bell number and this naive approach is obviously intractable. In this section, we provide an effective algorithm to partition a pivot set. Given a query workload $\mathcal{Q}$, our algorithm is based on the cost of evaluating queries in $\mathcal{Q}$ on a partition. In this section, we assume that we have a cost function, $\operatorname{Cost}_{\mathcal{Q}}(S)$, which estimates the cost for evaluating queries in $\mathcal{Q}$ on the partition determined by a pivot set $S$. We will present the cost function in the next section.

Given a pivot set $S$, we partition $S$ as follows. We first divide $S$ into two partitions $S_{1}$ and $S_{2}$ based on the estimated cost of evaluating queries on each partition. We then divide each partition again in the same manner as the first partitioning. We stop partitioning if it determines that further partitioning brings no benefit. Algorithm 3 shows the partitioning algorithm. It starts from two empty partitions $S_{1}$ and $S_{2}$ (Line 1). For each token $w$ in the pivot set $S$, it compares the benefit of adding $w$ into $S_{1}$ with the benefit of adding $w$ into $S_{2}$ (Lines 2-6). It adds $w$ into $S_{1}$ if the former is bigger than the latter (Line 5), otherwise it adds $w$ into $S_{2}$ (Line 6). Note that the pivot set selection algorithm presented in the previous section makes tokens in the selected pivot set sorted in descending order of their document frequencies. Thus, the partitioning algorithm always processes high frequency tokens first. After assigning each token in $S$ to either $S_{1}$ or $S_{2}$, the algorithm decides if it divides $S$ into $S_{1}$ and $S_{2}$ (Lines 7-10). It abandons $S_{1}$ and $S_{2}$ and stops partitioning if the cost of $S$ is less than the sum of costs of $S_{1}$ and $S_{2}$ (Line 8), otherwise it continues to divide $S_{1}$ and $S_{2}$ recursively (Line 10).

We extend Algorithm 3 to exploit those tokens which are not contained in the pivot set (see Example 12). We will present the details using the following definitions.

Definition 9: An atomic pivot set is a pivot set that cannot be partitioned because the pivot set contains only one token

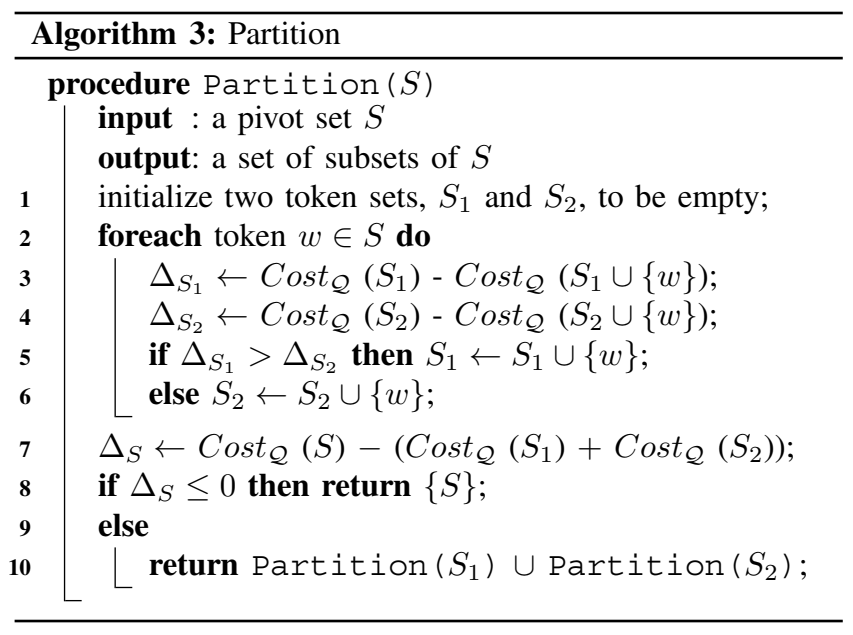

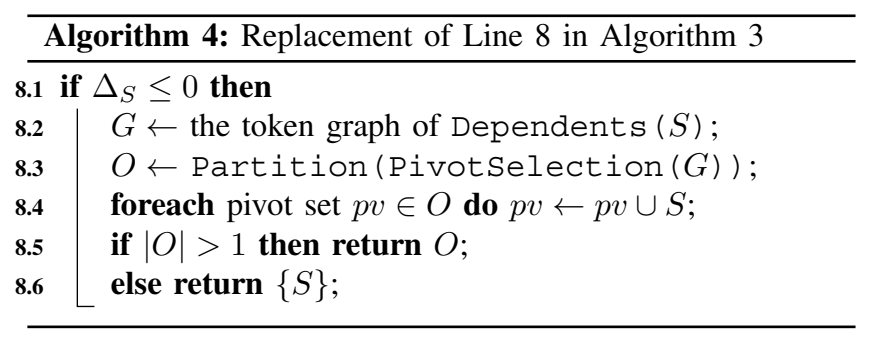

and/or Algorithm 3 determines that further partitioning of the pivot set brings no benefit.

Definition 10: Given a collection of records $C$, consider a pivot set $S$ and the partition $P$ determined by $S$. A token $w \notin S$ is a dependent token of the pivot set $S$ if and only if $d f_{P}(w)=d f_{C}(w)$ (see Example 13).

The basic idea of the extension is to continue partitioning by using dependent tokens of an atomic pivot set. We can effectively find dependent tokens of an atomic pivot set using the token graph according to the following lemma.

Lemma 3: Consider the pivot set $S$ of a partition $P$ of a collection of records. Using the pivot set $S$, we can calculate the document frequency of a token $w$ in $P$, which is denoted by $d f_{S}(w)$ as well as $d f_{P}(w)$, as:

$$
d f_{S}(w)=d f_{P}(w)=|R(w) \cap P|=\sum_{i=1}^{|S|}|R(w) \cap R(S[i])|,
$$

where $S[i]$ denotes the $i^{t h}$ token in $S$.

Algorithm 4 shows the extension of our partitioning algorithm. We only need to replace the if statement in Line 8 in Algorithm 3 with the if block in Lines 8.1-8.6 in Algorithm 4. If the current pivot set $S$ is atomic (Line 8.1), Algorithm 4 first finds dependent tokens of $S$ and extracts the token graph $G$ that consists of these dependent tokens from the token graph of the original collection (Line 8.2). It then selects a pivot set using $G$ and partition the pivot set recursively (Line 8.3). If the algorithm can further divide the atomic pivot set using its dependent tokens, it returns the result set of token sets (Line 8.5). Otherwise, it returns a set containing the atomic pivot set only (Line 8.6).

Example 13: Consider the collection depicted in Figure 3(b). Algorithm 2 selects the pivot set $\left\{w_{1}, w_{3}\right\}$. Algorithm 3 can at best divide the pivot set into $\left\{w_{1}\right\}$ and $\left\{w_{3}\right\}$. Algorithm 4 can further divide the large partition created by the atomic pivot set $\left\{w_{1}\right\}$ using the dependent tokens of $\left\{w_{1}\right\}$. The dependent tokens of $\left\{w_{1}\right\}$ are $w_{2}, w_{4}, w_{5}$, and $w_{7}$. The algorithm selects the pivot set $\left\{w_{2}, w_{4}\right\}$ from the dependent tokens and it can divide $\left\{w_{2}, w_{4}\right\}$ into $\left\{w_{2}\right\}$ and $\left\{w_{4}\right\}$. Therefore, the partitioning result can be $\left\{\left\{w_{2}\right\},\left\{w_{3}\right\},\left\{w_{4}\right\}\right\}$. 
For an atomic pivot set $S$, let $S^{\prime}$ be the pivot set made from dependent tokens of $S$. A problem with the extension is that $S^{\prime}$ may not cover all records covered by $S$ and as a result there could be orphan records which are not orphans without the extension.

Example 14: In Figure 3(b), the record $r_{10}$ belongs to the partition determined by $\left\{w_{1}\right\}$ after we partition the pivot set $\left\{w_{1}, w_{3}\right\}$ into $\left\{\left\{w_{1}\right\},\left\{w_{3}\right\}\right\}$. But if we further divide $\left\{w_{1}\right\}$ using its dependent tokens, $\left\{w_{1}\right\}$ is divided into $\left\{w_{2}\right\}$ and $\left\{w_{4}\right\}$ and we lose the partition determined by $\left\{w_{1}\right\}$. Because $r_{10}$ contains neither $w_{2}$ nor $w_{4}, r_{10}$ becomes an orphan record.

To prevent the phenomenon described in Example 14, we can insert $w_{1}$ into both $\left\{w_{2}\right\}$ and $\left\{w_{4}\right\}$ to make the partitioning result be $\left\{\left\{w_{1}, w_{2}\right\},\left\{w_{1}, w_{4}\right\},\left\{w_{3}\right\}\right\}$. In Algorithm 4, the foreach statement in Line 8.4 does this job. Although $r_{10}$ still does not decide its partition, we can make $r_{10}$ select a partition determined by either $w_{2}$ or $w_{4}$.

\section{Cost Function}

In this section, we present the cost function used in the previous section. Given a pivot set $S$ of a collection of record $C$, let $\operatorname{Cost}_{x, \delta}(S)$ be the estimated cost for evaluating a query $x$ with an overlap threshold $\delta$ on a partition determined by $S$. We can calculate $\operatorname{Cost}_{\mathcal{Q}}(S)$ by summing up $\operatorname{Cost}_{x, \delta}(S)$ for each query $(x, \delta) \in \mathcal{Q}$.

Let $x^{\prime}$ be $x \cap \mathcal{D}$, where $\mathcal{D}$ is the token dictionary of $C$. We propose a formula to calculate $\operatorname{Cost}_{x, \delta}(S)$ as:

$$
\operatorname{Cost}_{x, \delta}(S)=\left|x^{\prime}\right| \log \left|x^{\prime}\right|+\alpha \times\left|x^{\prime}\right| \times N_{x, \delta}(S),
$$

where $\alpha$ is a parameter dependent on the data set, and $N_{x, \delta}(S)$ is the estimated number of record ids in the prefix lists of $x^{\prime}$ in the partition of $S$.

The intuition behind the formula is as follows.

- To minimize the number of candidates, we sort the tokens in $x^{\prime}$ by their document frequencies in the partition of $S$. The cost for sorting is $\left|x^{\prime}\right| \log \left|x^{\prime}\right|$.

- The cost for merging the prefix lists of $x^{\prime}$ is approximately proportional to $N_{x, \delta}(S)$.

- The number of candidate records is approximately proportional to $N_{x, \delta}(S)$. Each candidate record searches the suffix lists of $x^{\prime}$. Because we use binary search, the cost for searching a suffix list is logarithmic in the search range, which is the size of the suffix list. However, a candidate does not search the whole search range of a suffix list because of the size filtering and the search range reduction caused by the sorted order of record ids (see Section III). We empirically observed that the cost for searching a suffix list was low and a constant value could be used as the cost in our cost function. Based on the observation, we simplify the verification cost to be proportional to $N_{x, \delta}(S) \times$ the number of the suffix lists of $x^{\prime}$. If we assume that overlap thresholds of queries do not vary widely, the number of the suffix lists of $x^{\prime}$ is approximately proportional to $\left|x^{\prime}\right|$. Thus, we

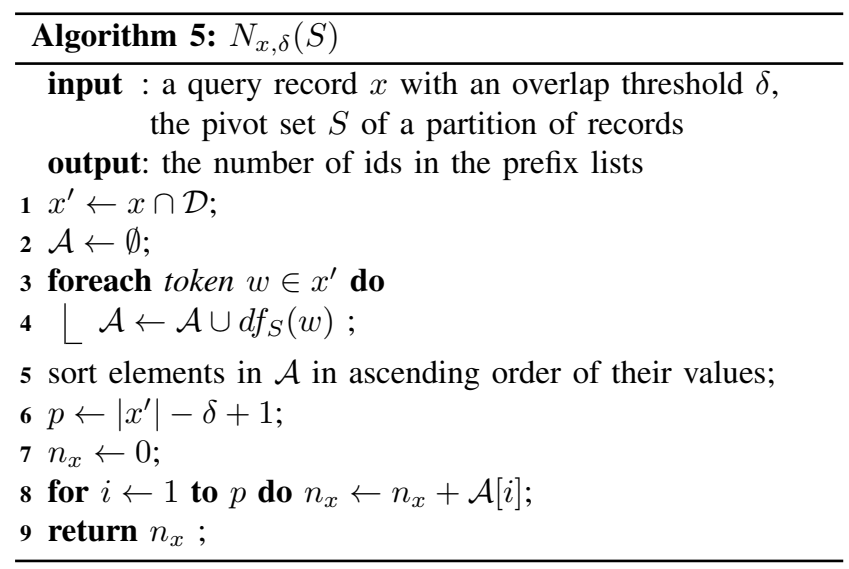

further simplify the verification cost to be proportional to $\left|x^{\prime}\right| \times N_{x, \delta}(S)$.

The parameter $\alpha$ in the cost function adjusts the estimated cost of merging the prefix lists, the number of candidates, and simplification of the cost for verifying candidates. It also reflects early termination in searching the suffix lists (see Section III). The parameter $\alpha$ plays a role of controlling the number of partitions to balance the trade-off between the benefit and the overhead of partitioning.

Now, we estimate $N_{x, \delta}(S)$, the number of record ids in the prefix list of $x^{\prime}$ in the partition determined by the pivot set $S$. Algorithm 5 calculates $N_{x, \delta}(S)$. The algorithm calculates the document frequency of each token in $x^{\prime}$ using Lemma 3 , and saves the frequency in an array $\mathcal{A}$ (Lines 2-4). Then it sorts the document frequencies in $\mathcal{A}$ in ascending order of their frequency values (Line 5). Finally, the algorithm accumulates the frequencies of the tokens in $\operatorname{prefix}_{\delta}\left(x^{\prime}\right)$ (Line 8).

Example 15: In Figure 1(c), consider the partition $P_{1}$ which is determined by the pivot set $S=\left\{w_{1}\right\}$. Given a query record $x=$ record("clerb", 2$)=\left\{w_{2}, w_{4}, w_{5}, r \mathrm{~b}\right\}$ with an overlap threshold $\delta=2$, we can calculate $N_{x, \delta}(S)$ as follows. We first identify $x^{\prime}=\left\{w_{2}, w_{4}, w_{5}\right\}$. For each token $w$ in $x^{\prime}$, we calculate $\left|R(w) \cap R\left(w_{1}\right)\right|$ and get the frequency array $\mathcal{A}=\left\{0_{\left(w_{2}\right)}, 2_{\left(w_{4}\right)}, 2_{\left(w_{5}\right)}\right\}$. Because the prefix size of the query is $2, N_{x, \delta}(S)$ is $0+2=2$.

$N_{x, \delta}(S)$ might not be the exact number of records in the prefix lists of $x$ due to the following reasons.

- Because the token graph of $C$ does not contain all tokens in $\mathcal{D}$, the number of records in some prefix lists may not be added to $N_{x, \delta}(S)$.

- If some prefix lists contain tokens from orphan records, $N_{x, \delta}(S)$ does not count those tokens in orphan records.

- The size filtering reduces the number of record ids in the prefix lists but we do not consider the size filtering when calculating $N_{x, \delta}(S)$.

The parameter $\alpha$ in the cost function is also used to reflect these factors. 


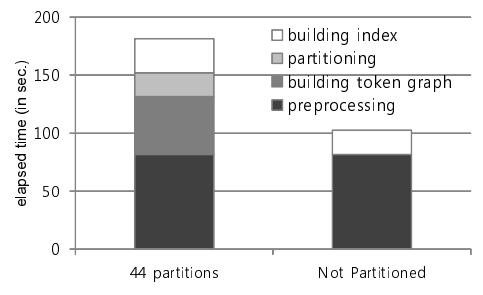

(a) Index build time

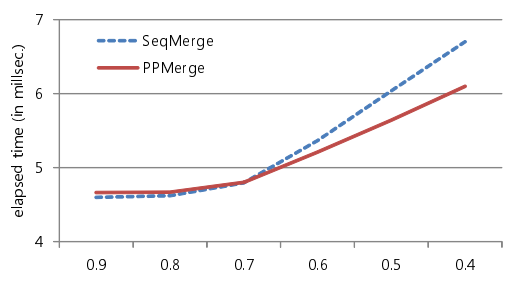

(b) parameter $\tau$ vs. query time

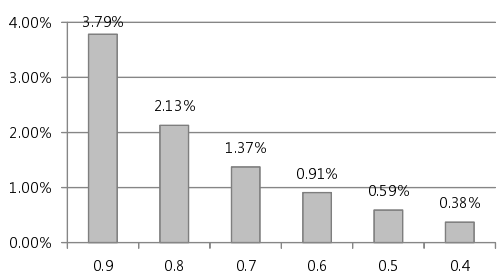

(c) parameter $\tau$ vs. $k /|\mathcal{D}|$ (\% of tokens)

Fig. 4. Index build time and results for token graph compaction parameter $\tau$

Although we simplify the cost function by putting together various factors into the parameter $\alpha$, we empirically show in Section VI that the parameter $\alpha$ is not very sensitive, which means that the range of appropriate numbers of partitions is wide.

\section{E. Relaxation of a Pivot Set}

Given a collection of records $C$, consider two tokens of $w_{1}$ and $w_{2}$ in $C$. Even if many records in $C$ contain either $w_{1}$ or $w_{2}$, a few records containing both tokens prevent these tokens from being used for constructing a pivot set. If there were many tokens such as $w_{1}$ and $w_{2}$, a pivot set might tend to be a small set, which is not adequate to make enough partitions. To make those tokens such as $w_{1}$ and $w_{2}$ be used in a pivot set, we can modify the definition of a pivot set as follows.

Definition 11: Given two sets of $s_{1}$ and $s_{2}$, let the Jaccard containment $J C\left(s_{1}, s_{2}\right)$ be $\frac{\left|s_{1} \cap s_{2}\right|}{\left|s_{1}\right|}$. A pivot set $S$ is a set of tokens such that for any two tokens $w_{i}$ and $w_{j}$ in $S$, $J C\left(R\left(w_{i}\right), R\left(w_{j}\right)\right) \leq \beta$ and $J C\left(R\left(w_{j}\right), R\left(w_{i}\right)\right) \leq \beta$, where $\beta$ is a positive real value close or equal to zero. Note that this definition is equivalent to Definition 4 if $\beta=0$.

Due to the relaxed definition of a pivot set, there can be some records having tokens from multiple pivot sets and those records cannot determine their partitions. The relaxation of a pivot set also makes Lemma 3 approximate. Because we find dependent tokens using Lemma 3, some dependent tokens are not actually dependent. This also causes some records to have tokens from multiple pivot sets and not to determine their partitions. However, the number of records that cannot determine their partitions is very small relative to the total number of records because the parameter $\beta$ in Definition 11 is close to zero. We can randomly assign records having tokens from multiple pivot sets to partitions determined by those pivot sets. Another problem is that Lemma 3 affects the cost function. However, we can easily handle the problem by tuning the parameter $\alpha$. Other minor changes caused by the relaxation (e.g. Definition 8) are straightforward and omitted in the interest of space.

\section{EXPERIMENTS}

We evaluated the performance of four merging algorithms: SeqMerge (SEQ), DivideSkip (DIV), PPMerge (PP), and ED-
Merge (ED) described in Section III. We used four real-world data sets: IMDB Actor and Movie (www.imdb.com), DBLP Author $^{3}$ (www.informatik.uni-trier.de/ ley/db), and Web Corpus (www.ldc.upenn.edu/Catalog, number LDC2006T13).

Some important statistics of the data sets are presented in Table I. We tokenized strings in the data sets into 3-grams. We selected 1000 strings uniformly at random from each data set to generate a workload for partitioning. We used Jaccard threshold 0.7 and edit distance 3 for each query in the workload for partitioning. All algorithms were implemented using GNU $\mathrm{C}++$ and ran on a iMac with $4 \mathrm{~GB}$ main memory and $2.66 \mathrm{GHz}$ Intel i5 $\mathrm{CPU}$ running Mac OSX operating system. We conducted experiments using another workload of randomly selected 1000 strings that was different from the workload used for building partitions. The inverted index was held in main memory.

TABLE I

DATA SETS USED IN EXPERIMENTS

\begin{tabular}{|c|r|r|c|}
\hline & \# of records & Avg. \# of tokens & \# of partitions \\
\hline IMDB Actor & $1,213,391$ & 16 & ED 28 Jaccard 12 \\
\hline IMDB Movie & $1,568,893$ & 19 & ED 18 Jaccard 12 \\
\hline DBLP Author & $2,948,929$ & 15 & ED 55 Jaccard 55 \\
\hline Web Corpus & $6,000,000$ & 21 & ED 54 Jaccard 85 \\
\hline
\end{tabular}

Index Build Time: Figure 4(a) shows the time for building index of the Web Corpus data set. The preprocessing time is for tokenizing and sorting the data set. It took about 170 seconds to build a partitioned inverted index, and it took about 100 seconds to build a normal inverted index. We observe that the partitioning phrase incurs about $70 \%$ of overhead in the index building time. However, an inverted index for search is generally built off-line and the build time is one-time cost, and thus we believe the overhead is a reasonable one.

Parameter Tuning: To tune parameters, we ran 1000 queries on the Web Corpus data set with the Jaccard similarity threshold 0.7 using the SeqMerge and PPMerge algorithms and measured the average time for evaluating a query. Figure 4(b) and (c) shows experimental results for the parameter $\tau$ used in building the token graph (see Section V(A)). We select

\footnotetext{
${ }^{3}$ This data set contains duplicated author names.
} 


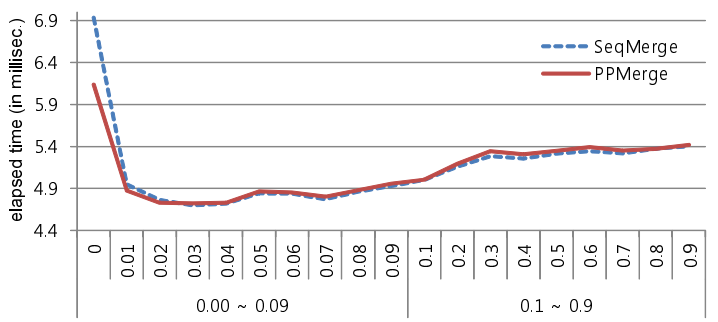

(a) Cost estimation parameter ( $\alpha$ ) vs. query time

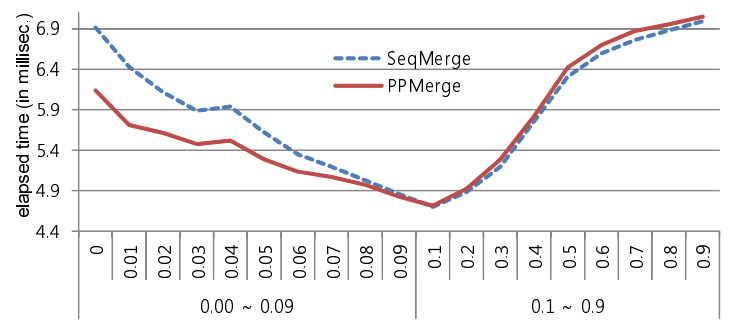

(b) Relaxation parameter $(\beta)$ vs. query time

Fig. 5. Experimental results for parameters $\alpha$ and $\beta$

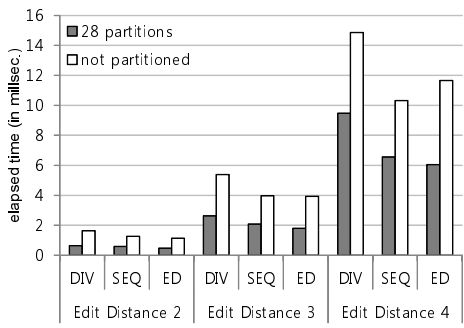

(a) Edit Distance, Actor, Time

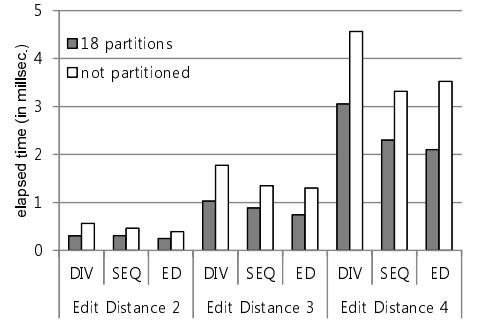

(d) Edit Distance, Movie, Time

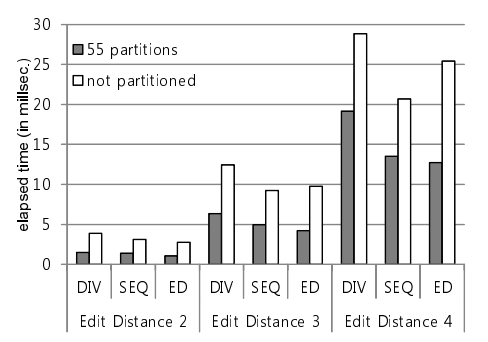

(g) Edit Distance, Author, Time

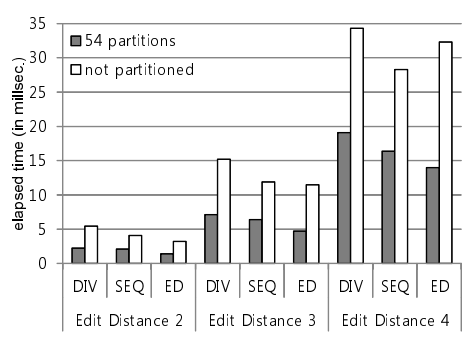

(j) Edist Distance, Corpus, Time

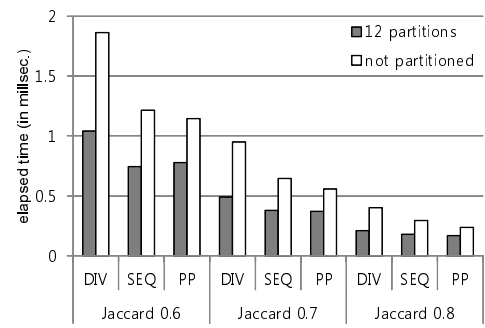

(b) Jaccard, Actor, Time

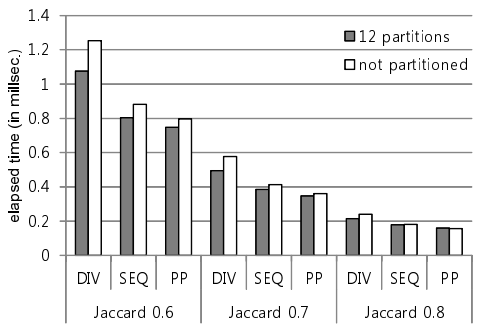

(e) Jaccard, Movie, Time

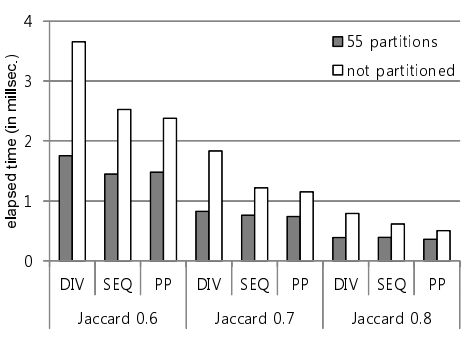

(h) Jaccard, Author, Time

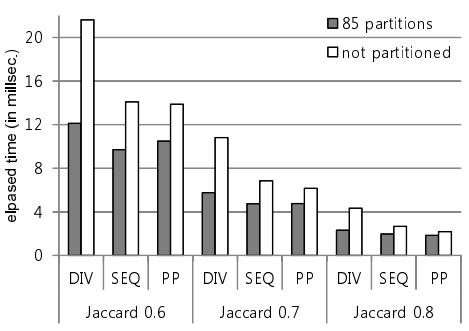

(k) Jaccard, Corpus, Time

Fig. 6. Experimental results

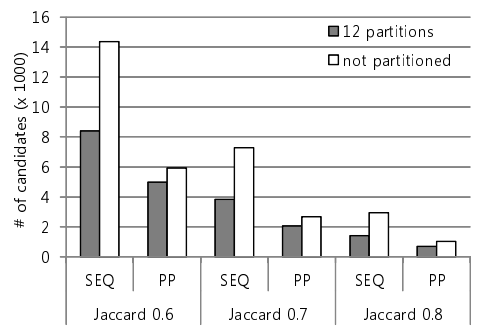

(c) Jaccard, Actor, Candidate size

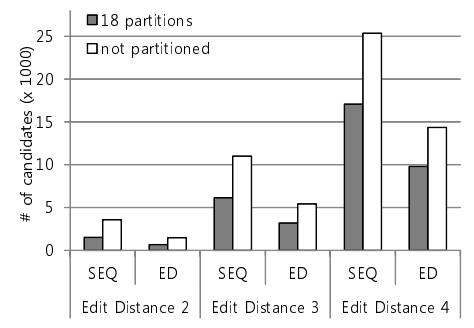

(f) Edit Distance, Movie, Candidate size

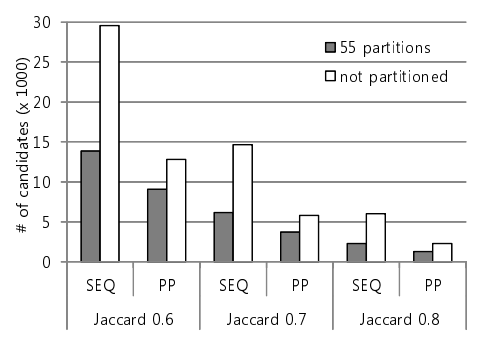

(i) Jaccard, Author, Candidate size

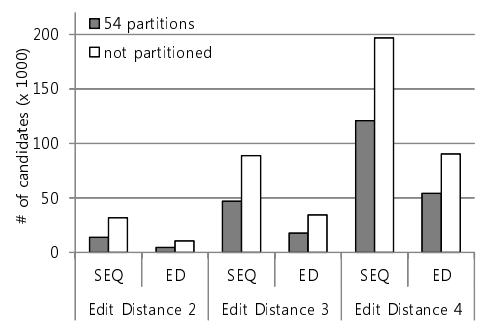

(1) Edit Distance, Corpus, Candidate size 
top- $k$ frequent tokens to build a token graph, and $\tau$ is the percentile threshold for sum of frequencies of selected top- $k$ tokens. While $\tau \geq 0.7$, the performance hardly degrades and we can see that the token graph can be built using only $1.37 \%$ of total number of tokens in the data set. In our experiments, we used 0.9 for the parameter $\tau$.

Figure 5(a) shows query times for the parameter $\alpha$ used in the cost function. The query time is optimized when the $\alpha$ value is less than 1.0. As we described in Section V(D), $\alpha$ reflects the size filtering on the prefix lists and is optimized to small value. An important observation is that $\alpha$ value is not very sensitive and exhibits good performance between 0.01 and 0.08 . We used 0.03 for the parameter $\alpha$ in our experiments.

Figure 5(b) shows query times for the parameter $\beta$ used in the relaxation of pivot sets described in Section $\mathrm{V}(\mathrm{E})$. The query time decreases until $\beta$ is 0.1 . Because the collection has many records, we cannot find a pivot set having enough tokens when $\beta$ is less than 0.03 . We can see that the performance sharply degrades when $\beta$ is larger than 0.2 and it justifies our observation on the pivot set partitioning. Based on this parameter tuning, we chose 0.1 for the parameter $\beta$.

Like [8], we added special symbols such as '\#' or ' $\$$ ' to each string as prefix and suffix before tokenizing the string when the similarity measure is the edit distance. Those prefix and suffix of strings in a data set enable a pivot set to have enough tokens and we do not need the relaxation of a pivot set. However, we found that a weak relaxation does not affect the performance and we used $\beta$ value tuned in Figure 5(b).

Query Time: Figure 6 compares the average execution times of a query with and without partitioning using various algorithms with edit distance and Jaccard similarity measures. We observed that the proposed technique clearly improves the performance of every algorithm in all settings. For example, for a query with edit distance 3 on the DBLP Author data set in Figure 6(g), EDMerge took $9.84 \mathrm{~ms}$ while it required just $4.27 \mathrm{~ms}$ after partitioning. The significant improvement can be explained by reduction in the number of candidates merged in the prefix lists. Figures 6(c), (f), (i), and (1) show the number of candidates generated. We can see that the proposed technique substantially reduces the number of candidates for all algorithms. In Figure 6(e), the improvement on the IMDB Movie data set is not as significant as that of other data sets. This is because it is hard to select a good pivot set from the data set. For the edit distance similarity measure, however, adding a prefix and a suffix to strings guarantees good pivot sets and apparent improvement was observed in the IMDB Movie data set as well as other data sets.

Note that we did not perform the final verification process when the similarity measure is the edit distance. If we do post-processing to remove false positives, EDMerge can be more efficient than other algorithms because it usually produces fewer false positives. We omit the comparison among the merge algorithms because it is rather orthogonal to the contribution of this paper.

\section{CONCLUSIONS}

In this paper, we presented a technique that exploits multiple token orderings by partitioning a collection of records. We demonstrated how to take advantage of token orderings to improve the performance of similarity searches. We proposed a novel concept of the pivot set and developed a partitioning algorithm. We evaluated the proposed technique using real data sets and showed that the proposed technique improves existing algorithms significantly.

\section{ACKNOWLEDGMENT}

This research was supported by Basic Science Research Program through the National Research Foundation of Korea (NRF) funded by the Ministry of Education, Science and Technology (No. 2011-0005411).

\section{REFERENCES}

[1] A. Arasu, V. Ganti, and R. Kaushik. Efficient exact set-similarity joins. In $V L D B$, pages 918-929, 2006.

[2] R. Bayardo, Y. Ma, and R. Srikant. Scaling up all-pairs similarity search. In WWW Conference, pages 131-140, 2007.

[3] A. Behm, S. Ji, C. Li, and J. Lu. Space-constrained gram-based indexing for efficient approximate string search. In ICDE, 2010.

[4] A. Behm, C. Li, and M. Carey. Answering approximate string queries on large data sets using external memory. In ICDE, pages 888-899, 2011.

[5] S. Chaudhuri, K. Ganjam, V. Ganti, and R. Motwani. Robust and efficient fuzzy match for online data cleaning. In SIGMOD Conference, pages 313-324, 2003.

[6] S. Chaudhuri, V. Ganti, and L. Gravano. Selectivity estimation for string predicates: Overcoming the underestimation problem. In ICDE, pages 227-238, 2004

[7] S. Chaudhuri, V. Ganti, and R. Kaushik. A primitive operator for similarity joins in data cleaning. In ICDE, page 5, 2006.

[8] L. Gravano, P. G. Ipeirotis, H. V. Jagadish, N. Koudas, S. Muthukrishnan, and D. Srivastava. Approximate string joins in a database (almost) for free. In $V L D B$, pages 491-500, 2001.

[9] N. Koudas, S. Sarawagi, and D. Srivastava. Record linkage: similarity measures and algorithms. In SIGMOD Tutorial, pages 802-803, 2005.

[10] C. Li, J. Lu, and Y. Lu. Efficient merging and filtering algorithms for approximate string searches. In ICDE, pages 257-266, 2008.

[11] C. Li, B. Wang, and X. Yang. VGRAM: Improving performance of approximate queries on string collections using variable-length grams. In $V L D B$, pages 303-314, 2007.

[12] J. Qin, W. Wang, Y. Lu, C. Xiao, and X. Lin. Efficient exact edit similarity query processing with asymmetric signature schemes. In SIGMOD Conference, pages 1033-1044, 2011.

[13] K. Ramasamy, J. M. Patel, R. Kaushik, and J. F. Naughton. Set containment joins: The good, the bad and the ugly. In $V L D B$, pages 351-362, 2000

[14] M. Sahami and T. D. Heilman. A web-based kernel function for measuring the similarity of short text snippets. In $W W W$, pages $377-386$, 2006.

[15] S. Sarawagi and A. Kirpal. Efficient set joins on similarity predicates. In SIGMOD Conference, pages 743-754, 2004.

[16] J. Wang, G. Li, and J. Feng. Fast-join: An efficient method for fuzzy token matching based string similarity join. In $I C D E$, pages $458-469$, 2011.

[17] C. Xiao, W. Wang, and X. Lin. Ed-join: an efficient algorithm for similarity joins with edit distance constraints. In $P V L D B$, pages 933944, 2008.

[18] C. Xiao, W. Wang, X. Lin, and H. Shang. Top-k set similarity joins. In ICDE, pages 916-927, 2009.

[19] C. Xiao, W. Wang, X. Lin, and J. Yu. Efficient similarity joins for near duplicate detection. In $W W W$, pages 131-140, 2008.

[20] X. Yang, B. Wang, and C. Li. Cost-based variable-length-gram selection for string collections to support approximate queries efficiently. In SIGMOD Conference, pages 353-364, 2008. 